1 Hacettepe Journal of Mathematics and Statistics

$\bigcap$ Volume 47 (1) (2018), $77-91$

\title{
New higher order iterative methods for solving nonlinear equations
}

\author{
Shuliang Huang*, Arif Rafiq ${ }^{\dagger}$, Muhammad Rizwan Shahzad ${ }^{\ddagger}$ and Faisal Ali ${ }^{\S}$
}

\begin{abstract}
In this paper, using the system of coupled equations involving an auxiliary function, we introduce some new efficient higher order iterative methods based on modified homotopy perturbation method. We study the convergence analysis and also present various numerical examples to demonstrate the validity and efficiency of our methods.
\end{abstract}

Keywords: Iterative methods, Nonlinear equations, Order of convergence, Auxiliary function, Modified homotopy perturbation method.

2000 AMS Classification: $65 \mathrm{Hxx}$

Received: 10.08.2016 Accepted: 18.02.2017 Doi : 10.15672/HJMS.2017.449

\section{Introduction}

Most of the arising problems in the fields of Mathematics and Engineering lead to the nonlinear equations. Scientists and engineers have devoted much attention to develop numerical techniques for solving nonlinear equations. Many iterative methods involving various techniques including Taylor series, decomposition, quadrature formulas, homotopy etc. have been proposed for the purpose, see $[1,3,4,8,9,11,12,17,22,23]$ and references therein. These methods are of quadratic, cubic and higher order convergence.

\footnotetext{
*Department of Mathematics, Chuzhou University, Chuzhou, China

Email : shulianghuang@163.com

${ }^{\dagger}$ Department of Mathematics and Statistics, Virtual University of Pakistan, Lahore 54000, Pakistan.

Email : aarafiq@gmail.com

${ }^{\ddagger}$ Centre for Advanced Studies in Pure and Applied Mathematics, Bahauddin Zakariya University, Multan 60800, Pakistan

Email : h.rizwanshahzad@gmail.com

${ }^{\S}$ Centre for Advanced Studies in Pure and Applied Mathematics, Bahauddin Zakariya University, Multan 60800, Pakistan

Email : faisalali@bzu.edu.pk ศ
} 
1.1. Definition. Let $\alpha$ be a simple zero of the nonlinear equation $f(x)=0, x_{n}$ the nth approximation to $\alpha$. If there exist a number $p \geqslant 1$ and a constant $c \neq 0$ such that $\lim _{n \rightarrow \infty} \frac{\left|x_{n+1}-\alpha\right|}{\left|x_{n}-\alpha\right|^{p}}=c$, then $\mathrm{p}$ is called the order of convergence of the method. The number $p^{\frac{1}{k}}$ is called the efficiency index of the method, where $\mathrm{k}$ is the number of new function evaluations required per iteration.

The following optimal fourth order iterative method was introduced by Chun [5].

$$
\begin{aligned}
& x_{n+1}^{*}=x_{n}-\frac{f\left(x_{n}\right)}{f^{\prime}\left(x_{n}\right)} \\
& x_{n+1}=x_{n+1}^{*}-\frac{f\left(x_{n}\right)+2 f\left(x_{n+1}^{*}\right)}{f\left(x_{n}\right)} \frac{f\left(x_{n+1}^{*}\right)}{f^{\prime}\left(x_{n}\right)}
\end{aligned}
$$

In 2007, Chun [8] also constructed the following fifth order method:

$$
x_{n+1}=x_{n+1}^{*}-\frac{f^{\prime}\left(x_{n+1}^{*}\right)+3 f^{\prime}\left(x_{n}\right)}{5 f^{\prime}\left(x_{n+1}^{*}\right)-f^{\prime}\left(x_{n}\right)} \frac{f\left(x_{n+1}^{*}\right)}{f^{\prime}\left(x_{n}\right)}
$$

Ostrovski [20] constructed the following optimal fourth order method:

$$
x_{n+1}=x_{n}-\frac{f\left(x_{n}\right)-f\left(x_{n+1}^{*}\right)}{f\left(x_{n}\right)-2 f\left(x_{n+1}^{*}\right)} \frac{f\left(x_{n}\right)}{f^{\prime}\left(x_{n}\right)}
$$

In 1994 Argyros et.al. [2] also introduced an optimal fourth order convergent method:

$$
\begin{aligned}
y_{n} & =x_{n}-\frac{2}{3} \frac{f\left(x_{n}\right)}{f^{\prime}\left(x_{n}\right)} \\
x_{n+1} & =x_{n}-\frac{1}{2} \frac{3 f^{\prime}\left(y_{n}\right)+f^{\prime}\left(x_{n}\right)}{3 f^{\prime}\left(y_{n}\right)-f^{\prime}\left(x_{n}\right)} \frac{f\left(x_{n}\right)}{f^{\prime}\left(x_{n}\right)}
\end{aligned}
$$

He [10] first introduced the Homotopy perturbation method (HPM) in 1999. Further, He modified his method in different ways $[12,13,14,15,16]$. Afterword, HPM has been extensively used by researchers in order to solve linear and nonlinear equations. In 2007, Golbabai and Javidi [7] introduced a third order iterative method for solving nonlinear equations using modified HPM. Later, Javidi [18] constructed the following method with convergence order five:

$$
x_{n+1}=x_{n+1}^{*}-\frac{f\left(x_{n+1}^{*}\right)}{f^{\prime}\left(x_{n+1}^{*}\right)}-\frac{1}{2}\left(\frac{f\left(x_{n+1}^{*}\right)}{f^{\prime}\left(x_{n+1}^{*}\right)}\right)^{2} \frac{f^{\prime \prime}\left(x_{n+1}^{*}\right)}{f^{\prime}\left(x_{n}\right)} .
$$

In 2011, Rafiullah [21] proposed a fifth order convergent method using modified HPM:

$$
x_{n+1}=x_{n+1}^{*}-\frac{f\left(x_{n+1}^{*}\right)}{f^{\prime}\left(x_{n+1}^{*}\right)}-\frac{1}{2}\left(\frac{f\left(x_{n+1}^{*}\right)}{f^{\prime}\left(x_{n}\right)}\right)^{2} \frac{f^{\prime \prime}\left(x_{n+1}^{*}\right)}{f^{\prime}\left(x_{n+1}^{*}\right)} .
$$

Recently, Shah and Noor [22] have proposed the following new method using auxiliary function and decomposition technique due to Daftardar-Gejji and Jafari [6] to find the solution of nonlinear equations;

$$
\begin{aligned}
y_{n} & =x_{n}-\frac{f\left(x_{n}\right)}{\left[f^{\prime}\left(x_{n}\right)-\beta f\left(x_{n}\right)\right]}, \\
z_{n} & =y_{n}-\frac{f\left(y_{n}\right)}{\left[f^{\prime}\left(x_{n}\right)-\beta f\left(x_{n}\right)\right]}, \\
x_{n+1} & =z_{n}-\frac{f\left(z_{n}\right)}{\left[f^{\prime}\left(x_{n}\right)-\beta f\left(x_{n}\right)\right]}, n=0,1,2 \ldots .
\end{aligned}
$$

We note that the efficiency indices of the methods defined in equations $(1.1)-(1.7)$ are $4^{\frac{1}{3}}, 5^{\frac{1}{4}}, 4^{\frac{1}{3}}, 4^{\frac{1}{3}}, 5^{\frac{1}{5}}, 5^{\frac{1}{5}}$ and $4^{\frac{1}{4}}$, respectively. 
In the present paper, we construct some new iterative methods with convergence order four and five for solving nonlinear equations using an auxiliary function and based on modified HPM [7]. The performance of our proposed methods has been revealed through a comparative study with some known methods by considering some test examples.

\section{Iterative Methods}

Consider the nonlinear equation

$$
f(x)=0 .
$$

Let $\alpha$ be a simple zero of equation (2.1) and $\gamma$ the initial guess sufficiently close to $\alpha$. Assume that $g(x)$ is the auxiliary function, such that

$$
f(x) g(x)=0 .
$$

Using Taylor series, we write nonlinear equation (2.2) in the form of coupled system as follows[22]:

$$
f(\gamma) g(\gamma)+\left[f^{\prime}(\gamma) g(\gamma)+f(\gamma) g^{\prime}(\gamma)\right](x-\gamma)+h(x)=0,
$$

where

$$
h(x)=f(x) g(\gamma)-f(\gamma) g(\gamma)-\left[f^{\prime}(\gamma) g(\gamma)+f(\gamma) g^{\prime}(\gamma)\right](x-\gamma)
$$

Equation (2.3) can be rewritten as:

$$
x=\gamma-\frac{f(\gamma) g(\gamma)}{\left[f^{\prime}(\gamma) g(\gamma)+f(\gamma) g^{\prime}(\gamma)\right]}-\frac{h(x)}{\left[f^{\prime}(\gamma) g(\gamma)+f(\gamma) g^{\prime}(\gamma)\right]}
$$

We write equation (2.5) in the following way

$$
x=c+N(x),
$$

where

$$
c=\gamma-\frac{f(\gamma) g(\gamma)}{\left[f^{\prime}(\gamma) g(\gamma)+f(\gamma) g^{\prime}(\gamma)\right]}
$$

and

$$
N(x)=-\frac{h(x)}{\left[f^{\prime}(\gamma) g(\gamma)+f(\gamma) g^{\prime}(\gamma)\right]} .
$$

Now, we construct a homotopy[7], $\Psi(x, \eta):(\mathbb{R} \times[0,1]) \times \mathbb{R} \rightarrow \mathbb{R}$ for equation (2.6), which satisfies

$$
\Psi(x, \eta)=x-c-\eta N(x)=0, \quad x \in \mathbb{R}, \eta \in[0,1],
$$

where $\eta$ is an embedding parameter. The trivial problem

$$
\Psi(x, 0)=x-c=0
$$

is incessantly deformed to the original problem

$$
\Psi(x, 1)=x-c-N(x)=0,
$$


as the embedding parameter $\eta$ monotonically increases from zero to unity. The modified HPM uses the embedding parameter $\eta$ as an expanding parameter to obtain [10]:

(2.12) $x=x_{0}+\eta x_{1}+\eta^{2} x_{2}+\ldots$.

Therefore, the approximate solution of equation (2.1) can readily be obtained as:

$$
x=\lim _{\eta \rightarrow 1} x=x_{0}+x_{1}+x_{2}+\ldots .
$$

It has been proved that the series $(2.13)$ is convergent [10].

Using the Taylor series expansion of $N(x)$ about $x_{0}$ and applying the modified HPM to equation (2.1), we can write equation (2.6) as

$$
\begin{aligned}
& x-c-\eta\left\{N\left(x_{0}\right)+\left(x-x_{0}\right) \frac{N^{\prime}\left(x_{0}\right)}{1 !}\right. \\
& \left.+\left(x-x_{0}\right)^{2} \frac{N^{\prime \prime}\left(x_{0}\right)}{2 !}+\ldots\right\}=0 .
\end{aligned}
$$

Substituting equation (2.12) into equation (2.14), we get

$$
\begin{aligned}
& x_{0}+\eta x_{1}+\eta^{2} x_{2}+\ldots-c- \\
& \eta\left\{N\left(x_{0}\right)+\left(x_{0}+\eta x_{1}+\eta^{2} x_{2}+\ldots-x_{0}\right) \frac{N^{\prime}\left(x_{0}\right)}{1 !}\right. \\
& \left.+\left(x_{0}+\eta x_{1}+\eta^{2} x_{2}+\ldots-x_{0}\right)^{2} \frac{N^{\prime \prime}\left(x_{0}\right)}{2 !}+\ldots\right\}=0 .
\end{aligned}
$$

Comparing alike powers of $\eta$ on both sides, we have

$$
\begin{aligned}
\eta^{0} & : \quad x_{0}-c=0, \\
\eta^{1} & : \quad x_{1}-N\left(x_{0}\right)=0, \\
\eta^{2} & : \quad x_{2}-x_{1} N^{\prime}\left(x_{0}\right)=0, \\
\eta^{3} & : \quad x_{3}-x_{2} N^{\prime}\left(x_{0}\right)-\frac{1}{2} x_{1}^{2} N^{\prime \prime}\left(x_{0}\right)=0 .
\end{aligned}
$$

From equations (2.7) and (2.16), we obtain

$$
x_{0}=c=\gamma-\frac{f(\gamma) g(\gamma)}{\left[f^{\prime}(\gamma) g(\gamma)+f(\gamma) g^{\prime}(\gamma)\right]} .
$$

Thus

(2.21) $\quad x \approx x_{0}=\gamma-\frac{f(\gamma) g(\gamma)}{\left[f^{\prime}(\gamma) g(\gamma)+f(\gamma) g^{\prime}(\gamma)\right]}$.

The above relation enables us to suggest the following iteration process.

2.1. Algorithm. For a given $x_{0}$, compute the approximate solution $x_{n+1}$, by the following iterative scheme:

$(2.22) \quad x_{n+1}=x_{n}-\frac{f\left(x_{n}\right) g\left(x_{n}\right)}{\left[f^{\prime}\left(x_{n}\right) g\left(x_{n}\right)+f\left(x_{n}\right) g^{\prime}\left(x_{n}\right)\right]}, \quad n=0,1,2, \ldots$.

The iterative method defined in equation (2.22) has also been introduced by $\mathrm{He}[15]$ and Noor[19] for generating various iterative methods for solving nonlinear equations.

From equations (2.4) and (2.20), it can easily be obtained that

$$
h\left(x_{0}\right)=f\left(x_{0}\right) g(\gamma)
$$


Using equations (2.4), (2.8) and (2.20), we get

$$
\begin{aligned}
& N\left(x_{0}\right)=-\frac{f\left(x_{0}\right) g(\gamma)}{\left[f^{\prime}(\gamma) g(\gamma)+f(\gamma) g^{\prime}(\gamma)\right]}, \\
& N^{\prime}\left(x_{0}\right)=1-\frac{f^{\prime}\left(x_{0}\right) g(\gamma)}{\left[f^{\prime}(\gamma) g(\gamma)+f(\gamma) g^{\prime}(\gamma)\right]},
\end{aligned}
$$

and

$$
N^{\prime \prime}\left(x_{0}\right)=-\frac{f^{\prime \prime}\left(x_{0}\right) g(\gamma)}{\left[f^{\prime}(\gamma) g(\gamma)+f(\gamma) g^{\prime}(\gamma)\right]} .
$$

Using equations $(2.17),(2.20)$ and $(2.24)$, we obtain

$$
x \approx x_{0}+x_{1}=\gamma-\frac{f(\gamma) g(\gamma)}{\left[f^{\prime}(\gamma) g(\gamma)+f(\gamma) g^{\prime}(\gamma)\right]}-\frac{f\left(x_{0}\right) g(\gamma)}{\left[f^{\prime}(\gamma) g(\gamma)+f(\gamma) g^{\prime}(\gamma)\right]}
$$

This formulation allows us the following recurrence relation for solving nonlinear equations.

2.2. Algorithm. For a given $x_{0}$, compute the approximate solution $x_{n+1}$ by the following iterative scheme:

$$
\begin{aligned}
x_{n+1} & =y_{n}-\frac{f\left(y_{n}\right) g\left(x_{n}\right)}{\left[f^{\prime}\left(x_{n}\right) g\left(x_{n}\right)+f\left(x_{n}\right) g^{\prime}\left(x_{n}\right)\right]}, \quad n=0,1,2, \ldots \\
y_{n} & =x_{n}-\frac{f\left(x_{n}\right) g\left(x_{n}\right)}{\left[f^{\prime}\left(x_{n}\right) g\left(x_{n}\right)+f\left(x_{n}\right) g^{\prime}\left(x_{n}\right)\right]} .
\end{aligned}
$$

The iterative method defined in equation (2.28) has also been introduced by shah and Noor[22] .

Now, using equations $(2.18),(2.24)$ and $(2.25)$, we get

$$
\begin{aligned}
x_{2} & =N\left(x_{0}\right) N^{\prime}\left(x_{0}\right) \\
& =-\frac{f\left(x_{0}\right) g(\gamma)}{\left[f^{\prime}(\gamma) g(\gamma)+f(\gamma) g^{\prime}(\gamma)\right]}+\frac{f\left(x_{0}\right) f^{\prime}\left(x_{0}\right) g^{2}(\gamma)}{\left[f^{\prime}(\gamma) g(\gamma)+f(\gamma) g^{\prime}(\gamma)\right]^{2}} .
\end{aligned}
$$

Thus, from equations (2.27) and (2.29), we have

$$
\begin{aligned}
x \approx & x_{0}+x_{1}+x_{2} \\
= & \gamma-\frac{f(\gamma) g(\gamma)}{\left[f^{\prime}(\gamma) g(\gamma)+f(\gamma) g^{\prime}(\gamma)\right]}-2 \frac{f\left(x_{0}\right) g(\gamma)}{\left[f^{\prime}(\gamma) g(\gamma)+f(\gamma) g^{\prime}(\gamma)\right]} \\
& +\frac{f\left(x_{0}\right) f^{\prime}\left(x_{0}\right) g^{2}(\gamma)}{\left[f^{\prime}(\gamma) g(\gamma)+f(\gamma) g^{\prime}(\gamma)\right]^{2}} .
\end{aligned}
$$

This formulation allows us the following recurrence relation for solving nonlinear equations.

2.3. Algorithm. For a given $x_{0}$, compute the approximate solution $x_{n+1}$ by the following iterative scheme:

$$
\begin{aligned}
x_{n+1} & =y_{n}-2 \frac{f\left(y_{n}\right) g\left(x_{n}\right)}{\left[f^{\prime}\left(x_{n}\right) g\left(x_{n}\right)+f\left(x_{n}\right) g^{\prime}\left(x_{n}\right)\right]}+\frac{f\left(y_{n}\right) f^{\prime}\left(y_{n}\right) g^{2}\left(x_{n}\right)}{\left[f^{\prime}\left(x_{n}\right) g\left(x_{n}\right)+f\left(x_{n}\right) g^{\prime}\left(x_{n}\right)\right]^{2}}, \\
y_{n} & =x_{n}-\frac{f\left(x_{n}\right) g\left(x_{n}\right)}{\left[f^{\prime}\left(x_{n}\right) g\left(x_{n}\right)+f\left(x_{n}\right) g^{\prime}\left(x_{n}\right)\right]} .
\end{aligned}
$$


Now, using equations $(2.19),(2.24),(2.25)$ and $(2.26)$, we get

$$
\begin{aligned}
x_{3}= & N\left(x_{0}\right) N^{\prime 2}\left(x_{0}\right)+\frac{1}{2} N^{2}\left(x_{0}\right) N^{\prime \prime}\left(x_{0}\right) \\
= & -\frac{f\left(x_{0}\right) g(\gamma)}{\left[f^{\prime}(\gamma) g(\gamma)+f(\gamma) g^{\prime}(\gamma)\right]}-\frac{f\left(x_{0}\right) f^{\prime 2}\left(x_{0}\right) g^{3}(\gamma)}{\left[f^{\prime}(\gamma) g(\gamma)+f(\gamma) g^{\prime}(\gamma)\right]^{3}} \\
& +2 \frac{f\left(x_{0}\right) f^{\prime}\left(x_{0}\right) g^{2}(\gamma)}{\left[f^{\prime}(\gamma) g(\gamma)+f(\gamma) g^{\prime}(\gamma)\right]^{2}}-\frac{1}{2} \frac{f^{2}\left(x_{0}\right) f^{\prime \prime}\left(x_{0}\right) g^{3}(\gamma)}{\left[f^{\prime}(\gamma) g(\gamma)+f(\gamma) g^{\prime}(\gamma)\right]^{3}} .
\end{aligned}
$$

Thus, from equations (2.30) and (2.31), we have

$$
\begin{aligned}
x \approx & x_{0}+x_{1}+x_{2}+x_{3} \\
= & \gamma-\frac{f(\gamma) g(\gamma)}{\left[f^{\prime}(\gamma) g(\gamma)+f(\gamma) g^{\prime}(\gamma)\right]}-3 \frac{f\left(x_{0}\right) g(\gamma)}{\left[f^{\prime}(\gamma) g(\gamma)+f(\gamma) g^{\prime}(\gamma)\right]} \\
& +3 \frac{f\left(x_{0}\right) f^{\prime}\left(x_{0}\right) g^{2}(\gamma)}{\left[f^{\prime}(\gamma) g(\gamma)+f(\gamma) g^{\prime}(\gamma)\right]^{2}}-\frac{f\left(x_{0}\right) f^{\prime 2}\left(x_{0}\right) g^{3}(\gamma)}{\left[f^{\prime}(\gamma) g(\gamma)+f(\gamma) g^{\prime}(\gamma)\right]^{3}} \\
& -\frac{1}{2} \frac{f^{2}\left(x_{0}\right) f^{\prime \prime}\left(x_{0}\right) g^{3}(\gamma)}{\left[f^{\prime}(\gamma) g(\gamma)+f(\gamma) g^{\prime}(\gamma)\right]^{3}} .
\end{aligned}
$$

This formulation allows us the following recurrence relation for solving nonlinear equations.

2.4. Algorithm. For a given $x_{0}$, compute the approximate solution $x_{n+1}$ by the following iterative scheme:

$$
\begin{aligned}
x_{n+1}= & y_{n}-3 \frac{f\left(y_{n}\right) g\left(x_{n}\right)}{\left[f^{\prime}\left(x_{n}\right) g\left(x_{n}\right)+f\left(x_{n}\right) g^{\prime}\left(x_{n}\right)\right]}+3 \frac{f\left(y_{n}\right) f^{\prime}\left(y_{n}\right) g^{2}\left(x_{n}\right)}{\left[f^{\prime}\left(x_{n}\right) g\left(x_{n}\right)+f\left(x_{n}\right) g^{\prime}\left(x_{n}\right)\right]^{2}} \\
& -\frac{f\left(y_{n}\right) f^{\prime 2}\left(y_{n}\right) g^{3}\left(x_{n}\right)}{\left[f^{\prime}\left(x_{n}\right) g\left(x_{n}\right)+f\left(x_{n}\right) g^{\prime}\left(x_{n}\right)\right]^{3}}-\frac{1}{2} \frac{f^{2}\left(y_{n}\right) f^{\prime \prime}\left(y_{n}\right) g^{3}\left(x_{n}\right)}{\left[f^{\prime}\left(x_{n}\right) g\left(x_{n}\right)+f\left(x_{n}\right) g^{\prime}\left(x_{n}\right)\right]^{3}}, \\
y_{n}= & x_{n}-\frac{f\left(x_{n}\right) g\left(x_{n}\right)}{\left[f^{\prime}\left(x_{n}\right) g\left(x_{n}\right)+f\left(x_{n}\right) g^{\prime}\left(x_{n}\right)\right]} .
\end{aligned}
$$

The implementation of algorithm 4 requires the value of second derivative, which is difficult in some cases. In order to avoid second derivative, the following finite difference approximation of second derivative is often used :

$$
f^{\prime \prime}\left(y_{n}\right)=\frac{f^{\prime}\left(y_{n}\right)-f^{\prime}\left(x_{n}\right)}{y_{n}-x_{n}} .
$$

Thus we have the following algorithm.

2.5. Algorithm. For a given $x_{0}$, compute the approximate solution $x_{n+1}$ by the following iterative scheme:

$$
\begin{aligned}
x_{n+1}= & y_{n}-3 \frac{f\left(y_{n}\right) g\left(x_{n}\right)}{\left[f^{\prime}\left(x_{n}\right) g\left(x_{n}\right)+f\left(x_{n}\right) g^{\prime}\left(x_{n}\right)\right]} \\
& +3 \frac{f\left(y_{n}\right) f^{\prime}\left(y_{n}\right) g^{2}\left(x_{n}\right)}{\left[f^{\prime}\left(x_{n}\right) g\left(x_{n}\right)+f\left(x_{n}\right) g^{\prime}\left(x_{n}\right)\right]^{2}}-\frac{f\left(y_{n}\right) f^{\prime 2}\left(y_{n}\right) g^{3}\left(x_{n}\right)}{\left[f^{\prime}\left(x_{n}\right) g\left(x_{n}\right)+f\left(x_{n}\right) g^{\prime}\left(x_{n}\right)\right]^{3}} \\
& -\frac{1}{2} \frac{f^{2}\left(y_{n}\right) g^{3}\left(x_{n}\right)}{\left[f^{\prime}\left(x_{n}\right) g\left(x_{n}\right)+f\left(x_{n}\right) g^{\prime}\left(x_{n}\right)\right]^{3}}\left(\frac{f^{\prime}\left(y_{n}\right)-f^{\prime}\left(x_{n}\right)}{y_{n}-x_{n}}\right), \\
y_{n}= & x_{n}-\frac{f\left(x_{n}\right) g\left(x_{n}\right)}{\left[f^{\prime}\left(x_{n}\right) g\left(x_{n}\right)+f\left(x_{n}\right) g^{\prime}\left(x_{n}\right)\right]} .
\end{aligned}
$$

It is clear that for different choices of the auxiliary function $g(x)$, several iterative methods with higher order convergence can be developed from the main iterative schemes established in this paper i.e. algorithm 3 and 5 . The contribution of the auxiliary 
function is the attractiveness of the modification of this technique. Proper selection of this auxiliary function converts the main recurrence relations in diversified form for best implementation for obtaining the solution of nonlinear equations.

To convey the basic idea, we consider the auxiliary function $g(x)=e^{-\beta x}$. Thus from Algorithm 3 and Algorithm 5, we obtain the following iterative methods for solving nonlinear equations.

2.6. Algorithm. For a given $x_{0}$, compute the approximate solution $x_{n+1}$ by the following iterative scheme:

$$
\begin{aligned}
x_{n+1} & =y_{n}-2 \frac{f\left(y_{n}\right)}{\left[f^{\prime}\left(x_{n}\right)-\beta f\left(x_{n}\right)\right]}+\frac{f\left(y_{n}\right) f^{\prime}\left(y_{n}\right)}{\left[f^{\prime}\left(x_{n}\right)-\beta f\left(x_{n}\right)\right]^{2}}, \\
y_{n} & =x_{n}-\frac{f\left(x_{n}\right)}{\left[f^{\prime}\left(x_{n}\right)-\beta f\left(x_{n}\right)\right]} .
\end{aligned}
$$

2.7. Algorithm. For a given $x_{0}$, compute the approximate solution $x_{n+1}$ by the following iterative scheme:

$$
\begin{aligned}
x_{n+1}= & y_{n}-3 \frac{f\left(y_{n}\right)}{\left[f^{\prime}\left(x_{n}\right)-\beta f\left(x_{n}\right)\right]}+3 \frac{f\left(y_{n}\right) f^{\prime}\left(y_{n}\right)}{\left[f^{\prime}\left(x_{n}\right)-\beta f\left(x_{n}\right)\right]^{2}} \\
& -\frac{f\left(y_{n}\right) f^{\prime 2}\left(y_{n}\right)}{\left[f^{\prime}\left(x_{n}\right)-\beta f\left(x_{n}\right)\right]^{3}}-\frac{1}{2} \frac{f^{2}\left(y_{n}\right)}{\left[f^{\prime}\left(x_{n}\right)-\beta f\left(x_{n}\right)\right]^{3}}\left(\frac{f^{\prime}\left(y_{n}\right)-f^{\prime}\left(x_{n}\right)}{y_{n}-x_{n}}\right), \\
y_{n}= & x_{n}-\frac{f\left(x_{n}\right)}{\left[f^{\prime}\left(x_{n}\right)-\beta f\left(x_{n}\right)\right]} .
\end{aligned}
$$

To the best of our knowledge, algorithm 6 and 7 are new.

\section{Convergence Analysis}

In this section, convergence analysis of newly proposed algorithm is studied in the form of the following theorem.

3.1. Theorem. Assume that the function $f: I \subset \mathbb{R} \rightarrow \mathbb{R}$ for an open interval $I$ has a simple root $\alpha \in I$. Let $f(x)$ be sufficiently differentiable in the neighborhood of the root $\alpha$, then the convergence order of the methods defined by algorithm 3 and 5 are four and five respectively.

Proof. Let $\alpha$ be a simple zero of $f(x)$. Since $f$ is sufficiently differentiable, the Taylor series expansions of $f\left(x_{n}\right), f^{\prime}\left(x_{n}\right), g\left(x_{n}\right)$ and $g^{\prime}\left(x_{n}\right)$ about $\alpha$ are given by

$$
\begin{gathered}
f\left(x_{n}\right)=f^{\prime}(\alpha)\left\{e_{n}+c_{2} e_{n}^{2}+c_{3} e_{n}^{3}+c_{4} e_{n}^{4}+c_{5} e_{n}^{5}+O\left(e_{n}^{6}\right)\right\} \\
f^{\prime}\left(x_{n}\right)=f^{\prime}(\alpha)\left\{1+2 c_{2} e_{n}+3 c_{3} e_{n}^{2}+4 c_{4} e_{4}^{3}+5 c_{5} e_{n}^{4}+6 c_{6} e_{n}^{5}+O\left(e_{n}^{6}\right)\right\} \\
g\left(x_{n}\right)=\quad \\
\begin{array}{c}
(\alpha)+g^{\prime}(\alpha) e_{n}+\frac{g^{\prime \prime}(\alpha)}{2} e_{n}^{2}+\frac{g^{\prime \prime \prime}(\alpha)}{6} e_{n}^{3}+\frac{g^{(i v)}(\alpha)}{24} e_{n}^{4} \\
+\frac{g^{(v)}(\alpha)}{120} e_{n}^{5}+O\left(e_{n}^{6}\right)
\end{array}
\end{gathered}
$$

and

$$
\begin{aligned}
g^{\prime}\left(x_{n}\right)= & g^{\prime}(\alpha)+g^{\prime \prime}(\alpha) e_{n}+\frac{g^{\prime \prime \prime}(\alpha)}{2} e_{n}^{2}+\frac{g^{(i v)}(\alpha)}{6} e_{n}^{3}+\frac{g^{(v)}(\alpha)}{24} e_{n}^{4} \\
& +\frac{g^{(v i)}(\alpha)}{120} e^{5}+O\left(e_{n}^{6}\right),
\end{aligned}
$$


respectively, where $e_{n}=x_{n}-\alpha$ and $c_{j}=\frac{1}{j !} \frac{f^{(j)}(\alpha)}{f^{\prime}(\alpha)}, \quad j=2,3, \ldots$.

Using equations (3.1), (3.2), (3.3), and (3.4), we have

$$
\begin{aligned}
f\left(x_{n}\right) g\left(x_{n}\right)= & f^{\prime}(\alpha)\left\{g(\alpha) e_{n}+\left(c_{2} g(\alpha)+g^{\prime}(\alpha)\right) e_{n}^{2}+\left(\frac{1}{2} g^{\prime \prime}(\alpha)+c_{2} g^{\prime}(\alpha)\right.\right. \\
& \left.+c_{3} g(\alpha)\right) e_{n}^{3}+\left(\frac{1}{6} g^{\prime \prime \prime}(\alpha)+\frac{1}{2} c_{2} g^{\prime \prime}(\alpha)+c_{3} g^{\prime}(\alpha)+c_{4} g(\alpha)\right) e_{n}^{4} \\
& \left.+O\left(e_{n}^{5}\right)\right\},
\end{aligned}
$$

$$
\begin{aligned}
f^{\prime}\left(x_{n}\right) g\left(x_{n}\right)= & f^{\prime}(\alpha)\left\{g(\alpha)+\left(2 c_{2} g(\alpha)+g^{\prime}(\alpha)\right) e_{n}+\left(\frac{1}{2} g^{\prime \prime}(\alpha)+2 c_{2} g^{\prime}(\alpha)\right.\right. \\
& \left.+3 c_{3} g(\alpha)\right) e_{n}^{2}+\left(\frac{1}{6} g^{\prime \prime \prime}(\alpha)+c_{2} g^{\prime \prime}(\alpha)+3 c_{3} g^{\prime}(\alpha)+4 c_{4} g(\alpha)\right) e_{n}^{3} \\
& +\frac{1}{24} g^{(i v)}(\alpha)+\frac{1}{3} c_{2} g^{\prime \prime \prime}(\alpha)+\frac{3}{2} c_{3} g^{\prime \prime}(\alpha)+4 c_{4} g^{\prime}(\alpha) \\
& \left.\left.+5 c_{5} g(\alpha)\right) e_{n}^{4}+O\left(e_{n}^{5}\right)\right\}
\end{aligned}
$$

and

$$
\begin{aligned}
f\left(x_{n}\right) g^{\prime}\left(x_{n}\right)= & f^{\prime}(\alpha)\left\{g^{\prime}(\alpha) e_{n}+\left(c_{2} g^{\prime}(\alpha)+g^{\prime \prime}(\alpha)\right) e_{n}^{2}+\left(\frac{1}{2} g^{\prime \prime \prime}(\alpha)+c_{2} g^{\prime \prime}(\alpha)\right.\right. \\
& \left.+c_{3} g^{\prime}(\alpha)\right) e_{n}^{3}+\left(\frac{1}{6} g^{(i v)}(\alpha)+\frac{1}{2} c_{2} g^{\prime \prime \prime}(\alpha)+c_{3} g^{\prime \prime}(\alpha)+c_{4} g^{\prime}(\alpha)\right) e_{n}^{4} \\
& \left.+O\left(e_{n}^{5}\right)\right\} .
\end{aligned}
$$

From equations (3.5), (3.6) and (3.7), we get

$$
\begin{aligned}
\frac{f\left(x_{n}\right) g\left(x_{n}\right)}{f^{\prime}\left(x_{n}\right) g\left(x_{n}\right)+f\left(x_{n}\right) g^{\prime}\left(x_{n}\right)}= & e_{n}-\left(c_{2}+\frac{g^{\prime}(\alpha)}{g(\alpha)}\right) e_{n}^{2}+\left(2 c_{2} \frac{g^{\prime}(\alpha)}{g(\alpha)}-2 c_{3}-\frac{g^{\prime \prime}(\alpha)}{g(\alpha)}\right. \\
& \left.+2 c_{2}^{2}+2\left(\frac{g^{\prime}(\alpha)}{g(\alpha)}\right)^{2}\right) e_{n}^{3}+\left(-\frac{1}{2} \frac{g^{\prime \prime \prime}(\alpha)}{g(\alpha)}+2 c_{2} \frac{g^{\prime \prime}(\alpha)}{g(\alpha)}\right. \\
& +4 c_{3} \frac{g^{\prime}(\alpha)}{g(\alpha)}-3 c_{4} \frac{7}{2} g^{\prime}(\alpha) \frac{g^{\prime \prime}(\alpha)}{(g(\alpha))^{2}}-5 c_{2}^{2} \frac{g^{\prime}(\alpha)}{g(\alpha)} \\
& \left.-5 c_{2}\left(\frac{g^{\prime}(\alpha)}{g(\alpha)}\right)^{2}+7 c_{2} c_{3}-4 c_{2}^{3}-4\left(\frac{g^{\prime}(\alpha)}{g(\alpha)}\right)^{3}\right) e_{n}^{4} \\
& +O\left(e_{n}^{5}\right) .
\end{aligned}
$$

Using equation (3.8), we get

$$
\begin{aligned}
y_{n}= & \alpha+\left(c_{2}+\frac{g^{\prime}(\alpha)}{g(\alpha)}\right) e_{n}^{2}+\left(2 c 3-2 c_{2} \frac{g^{\prime}(\alpha)}{g(\alpha)}+\frac{g^{\prime \prime}(\alpha)}{g(\alpha)}-2 c_{2}^{2}-2\left(\frac{g^{\prime}(\alpha)}{g(\alpha)}\right)^{2}\right) e_{n}^{3} \\
& +\frac{1}{2} \frac{g^{\prime \prime \prime}(\alpha)}{g(\alpha)}-2 c_{2} \frac{g^{\prime \prime}(\alpha)}{g(\alpha)}-4 c_{3} \frac{g^{\prime}(\alpha)}{g(\alpha)}+3 c_{4}-\frac{7}{2} \frac{g^{\prime}(\alpha) g^{\prime \prime}(\alpha)}{(g(\alpha))^{2}}+5 c_{2}^{2} \frac{g^{\prime}(\alpha)}{g(\alpha)} \\
& \left.+5 c_{2}\left(\frac{g^{\prime}(\alpha)}{g(\alpha)}\right)^{2}-7 c_{2} c_{3}+4 c_{2}^{3}+4\left(\frac{g^{\prime}(\alpha)}{g(\alpha)}\right)^{3}\right) e_{n}^{4}+O\left(e_{n}^{5}\right) .
\end{aligned}
$$


Taylor series expansions of $f\left(y_{n}\right)$ and $f^{\prime}\left(y_{n}\right)$ about $\alpha$ are given as:

$$
\begin{aligned}
f\left(y_{n}\right)= & f^{\prime}(\alpha)\left\{\left(c_{2}+\frac{g^{\prime}(\alpha)}{g(\alpha)}\right) e_{n}^{2}+\left(2 c_{3}-2 c_{2} \frac{g^{\prime}(\alpha)}{g(\alpha)}+\frac{g^{\prime \prime}(\alpha)}{g(\alpha)}-2 c_{2}^{2}-2\left(\frac{g^{\prime}(\alpha)}{g(\alpha)}\right)^{2}\right) e_{n}^{3}\right. \\
& +\left(\frac{1}{2} \frac{g^{\prime \prime \prime}(\alpha)}{g(\alpha)}-2 c_{2} \frac{g^{\prime \prime}(\alpha)}{g(\alpha)}-4 c_{3} \frac{g^{\prime}(\alpha)}{g(\alpha)}+3 c_{4}-\frac{7}{2} \frac{g^{\prime}(\alpha) g^{\prime \prime}(\alpha)}{(g(\alpha))^{2}}+7 c_{2}^{2} \frac{g^{\prime}(\alpha)}{g(\alpha)}\right. \\
& \left.\left.+6 c_{2}\left(\frac{g^{\prime}(\alpha)}{g(\alpha)}\right)^{2}-7 c_{2} c_{3}+5 c_{2}^{3}+4\left(\frac{g^{\prime}(\alpha)}{(g(\alpha)}\right)\right) e_{n}^{4}+O\left(e_{n}^{5}\right)\right\}
\end{aligned}
$$

and

$$
\begin{aligned}
f^{\prime}\left(y_{n}\right)= & f^{\prime}(\alpha)\left\{1+\left(2 c_{2}^{2}+2 c_{2} \frac{g^{\prime}(\alpha)}{g(\alpha)}\right) e_{n}^{2}+\left(4 c_{3} c_{2}-4 c_{2}^{2} \frac{g^{\prime}(\alpha)}{g(\alpha)}+2 c_{2} \frac{g^{\prime \prime}(\alpha)}{g(\alpha)}\right.\right. \\
& \left.-4 c_{2}^{3}-4 c_{2}\left(\frac{g^{\prime}(\alpha)}{g(\alpha)}\right)^{2}\right) e_{n}^{3}+\left(c_{2} \frac{g^{\prime \prime \prime}(\alpha)}{g(\alpha)}-4 c_{2}^{2} \frac{g^{\prime \prime}(\alpha)}{g(\alpha)}-2 c_{2} c_{3} \frac{g^{\prime}(\alpha)}{g(\alpha)}\right. \\
& +6 c_{2} c_{4}-7 c_{2} \frac{g^{\prime}(\alpha) g^{\prime \prime}(\alpha)}{(g(\alpha))^{2}}+10 c_{2}^{3} \frac{g^{\prime}(\alpha)}{g(\alpha)}+10 c_{2}^{2}\left(\frac{g^{\prime}(\alpha)}{g(\alpha)}\right)^{2}-11 c_{2}^{2} c_{3} \\
& \left.\left.+8 c_{2}^{4}+8 c_{2}\left(\frac{g^{\prime}(\alpha)}{g(\alpha)}\right)^{3}+3 c_{3}\left(\frac{g^{\prime}(\alpha)}{g(\alpha)}\right)^{2}\right) e_{n}^{4}+O\left(e_{n}^{5}\right)\right\},
\end{aligned}
$$

respectively. Taylor series expansion of $f\left(y_{n}\right) g(x n)$ using (3.3) and (3.10) is

$$
\begin{aligned}
f\left(y_{n}\right) g\left(x_{n}\right)= & \left(c_{2} g(\alpha)+g^{\prime}(\alpha)\right) e_{n}^{2}+\left(-c_{2} g^{\prime}(\alpha)-\frac{\left(g^{\prime}(\alpha)\right)^{2}}{g(\alpha)}+2 c_{3} g(\alpha)+g^{\prime \prime}(\alpha)\right. \\
& \left.-2 c_{2}^{2} g(\alpha)\right) e_{n}^{3}+\left(-\frac{3}{2} c_{2} g^{\prime \prime}(\alpha)-2 g^{\prime \prime}(\alpha) \frac{g^{\prime}(\alpha)}{g(\alpha)}+4 c_{2} \frac{\left(g^{\prime}(\alpha)\right)^{2}}{g(\alpha)}\right. \\
& -2 c_{3} g^{\prime}(\alpha)+5 c_{2}^{2} g^{\prime}(\alpha)+\frac{2\left(g^{\prime}(\alpha)\right)^{3}}{(g(\alpha))^{2}}+\frac{1}{2 g^{\prime \prime \prime}(\alpha)}+3 c_{4} g(\alpha) \\
& \left.-7 c_{2} c_{3} g(\alpha)+5 c_{2}^{3} g(\alpha)\right) e_{n}^{4}+O\left(e_{n}^{5}\right) .
\end{aligned}
$$

From equations (3.6), (3.7) and (3.12), we obtain

$$
\begin{aligned}
\frac{f\left(y_{n}\right) g\left(x_{n}\right)}{f^{\prime}\left(x_{n}\right) g\left(x_{n}\right)+f\left(x_{n}\right) g^{\prime}\left(x_{n}\right)}= & \left(c_{2}+\frac{g^{\prime}(\alpha)}{g(\alpha)}\right) e^{2}+\left(-5 c_{2} \frac{g^{\prime}(\alpha)}{g(\alpha)}-3\left(\frac{g^{\prime}(\alpha)}{g(\alpha)}\right)^{2}\right. \\
& \left.+2 c_{3}+3 \frac{g^{\prime \prime}(\alpha)}{g(\alpha)}-4 c_{2}^{2}\right) e^{3}+\left(-5 c_{2} \frac{g^{\prime \prime}(\alpha)}{g(\alpha)}\right. \\
& -\frac{11}{2} g^{\prime}(p) \frac{g^{\prime \prime}(\alpha)}{(g(\alpha))^{2}}+17 c_{2}\left(\frac{g^{\prime}(\alpha)}{g(\alpha)}\right)^{2}-9 c_{3} \frac{g^{\prime}(\alpha)}{g(\alpha)} \\
& +20 c_{2}^{2} \frac{g^{\prime}(\alpha)}{g(\alpha)}+8\left(\frac{g^{\prime}(\alpha)}{g(\alpha)}\right)^{3}+\frac{1}{2} \frac{g^{\prime \prime \prime}(\alpha)}{g(\alpha)}+3 c_{4} \\
& \left.+13 c_{2}^{3}-14 c_{2} c_{3}\right) e^{4}+O\left(e_{n}^{5}\right) .
\end{aligned}
$$


Using equations (3.3), (3.6), (3.7), (3.10) and (3.11), we have

$$
\begin{aligned}
\frac{f\left(y_{n}\right) f^{\prime}\left(y_{n}\right) g^{2}\left(x_{n}\right)}{\left[f^{\prime}\left(x_{n}\right) g\left(x_{n}\right)+f\left(x_{n}\right) g^{\prime}\left(x_{n}\right)\right]^{2}}= & \left(c_{2}+\frac{g^{\prime}(\alpha)}{g(\alpha)}\right) e^{2}+\left(-6 c_{2}^{2}-8 c_{2} \frac{g^{\prime}(\alpha)}{g(\alpha)}\right. \\
& \left.-4\left(\frac{g^{\prime}(\alpha)}{g(\alpha)}\right)^{2}+2 c_{3}+3 \frac{g^{\prime \prime}(\alpha)}{g(\alpha)}\right) e^{3}+\left(27 c_{2}^{3}\right. \\
& +45 c_{2}^{2} \frac{g^{\prime}(\alpha)}{g(\alpha)}+35 c_{2}\left(\frac{g^{\prime}(\alpha)}{g(\alpha)}\right)^{2}-21 c_{2} c_{3} \\
& -14 c_{3} \frac{g^{\prime}(\alpha)}{g(\alpha)}+13\left(\frac{g^{\prime}(\alpha)}{g(\alpha)}\right)^{3}-8 c_{2} \frac{g^{\prime \prime}(\alpha)}{g(\alpha)} \\
& \left.-\frac{15}{2} g^{\prime}(p) \frac{g^{\prime \prime}(\alpha)}{(g(\alpha))^{2}}+\frac{1}{2} \frac{g^{\prime \prime \prime}(\alpha)}{g(\alpha)}+3 c_{4}\right) e^{4}+O\left(e_{n}^{5}\right) .
\end{aligned}
$$

Thus, using equations (3.9), (3.13) and (3.14), the error term for Algorithm 3 can be obtained as

$$
e_{n+1}=\left(5 c_{2}^{3}+\left(\frac{g^{\prime}(\alpha)}{g(\alpha)}\right)^{3}+10 c_{2}^{2} \frac{g^{\prime}(\alpha)}{g(\alpha)}+6 c_{2}\left(\frac{g^{\prime}(\alpha)}{g(\alpha)}\right)^{2}\right) e^{4}+O\left(e^{5}\right) .
$$

Now using equations (3.3), (3.6), (3.7), (3.10) and (3.11), we obtain

$$
\begin{aligned}
\frac{f\left(y_{n}\right) f^{\prime 2}\left(y_{n}\right) g^{3}\left(x_{n}\right)}{\left[f^{\prime}\left(x_{n}\right) g\left(x_{n}\right)+f\left(x_{n}\right) g^{\prime}\left(x_{n}\right)\right]^{3}}= & \left(c 2+\frac{g^{\prime}(\alpha)}{g(\alpha)}\right) e^{2}+\left(-8 c_{2}^{2}-11 c_{2} \frac{g^{\prime}(\alpha)}{g(\alpha)}\right. \\
& \left.-5\left(\frac{g^{\prime}(\alpha)}{g(\alpha)}\right)^{2}+2 c_{3}+\frac{g^{\prime \prime}(\alpha)}{g(\alpha)}\right) e^{3}+\left(3 c_{4}\right. \\
& +\frac{1}{2} \frac{g^{\prime \prime \prime}(\alpha)}{g(\alpha)}+45 c_{2}^{3}+19\left(\frac{g^{\prime}(\alpha)}{g(\alpha)}\right)^{3}-11 c_{2} \frac{g^{\prime \prime}(\alpha)}{g(\alpha)} \\
& -19 c_{3} \frac{g^{\prime}(\alpha)}{g(\alpha)}-\frac{19}{2} g^{\prime}(\alpha) \frac{g^{\prime \prime}(\alpha)}{(g(\alpha))^{2}}+78 c_{2}^{2} \frac{g^{\prime}(\alpha)}{g(\alpha)} \\
& \left.+58 c_{2}\left(\frac{g^{\prime}(\alpha)}{g(\alpha)}\right)^{2}-28 c_{2} c_{3}\right) e^{4}+O\left(e_{n}^{5}\right)
\end{aligned}
$$

and

$$
\frac{f^{2}\left(y_{n}\right) g^{3}\left(x_{n}\right)}{\left[f^{\prime}\left(x_{n}\right) g\left(x_{n}\right)+f\left(x_{n}\right) g^{\prime}\left(x_{n}\right)\right]^{3}}=\left(c_{2}^{2}+2 c_{2} \frac{g^{\prime}(\alpha)}{g(\alpha)}+\left(\frac{g^{\prime}(\alpha)}{g(\alpha)}\right)^{2}\right) e^{4}+O\left(e_{n}^{5}\right) .
$$

Using (3.2), (3.9), (3.11) and (3.16), we have

$$
\begin{array}{ll}
\frac{f^{2}\left(y_{n}\right) g^{3}\left(x_{n}\right)}{\left[f^{\prime}\left(x_{n}\right) g\left(x_{n}\right)+f\left(x_{n}\right) g^{\prime}\left(x_{n}\right)\right]^{3}}\left(\frac{f^{\prime}\left(y_{n}\right)-f^{\prime}\left(x_{n}\right)}{y_{n}-x_{n}}\right)= & \left(2 c_{2}^{3}+4 c_{2}^{2} \frac{g^{\prime}(\alpha)}{g(\alpha)}\right. \\
3.17) & \left.+2 c_{2}\left(\frac{g^{\prime}(\alpha)}{g(\alpha)}\right)^{2}\right) e^{4}+O\left(e_{n}^{5}\right)
\end{array}
$$


Thus from equations (3.9), (3.13) , (3.14), (3.15) and (3.17) the error term for Algorithm 5 is given by

$$
\begin{aligned}
e_{n+1}= & \left(14 c_{2}^{4}+\left(\frac{g^{\prime}(\alpha)}{g(\alpha)}\right)^{4}-3 c_{2} c_{3} \frac{g^{\prime}(\alpha)}{g(\alpha)}-\frac{3}{2} c_{3}\left(\frac{g^{\prime}(\alpha)}{g(\alpha)}\right)^{2}+35 c_{2}^{3} \frac{g^{\prime}(\alpha)}{g(\alpha)}\right. \\
& \left.+30 c_{2}^{2}\left(\frac{g^{\prime}(\alpha)}{g(\alpha)}\right)^{2}+10 c_{2}\left(\frac{g^{\prime}(\alpha)}{g(\alpha)}\right)^{3}-\frac{3}{2} c_{2}^{2} c_{3}\right) e_{n}^{5}+O\left(e_{n}^{6}\right) .
\end{aligned}
$$

This completes the proof.

It is noticable that the efficiency indices of the methods developed here and formulated in algorithm 6 and 7 are $4^{\frac{1}{4}}$ and $5^{\frac{1}{4}}$, respectively.

\section{Numerical Examples}

Table-1 demonstrates the comparison of Algorithm 6 (RM1) with standard Newton's method (NM) and some known methods with convergence order 4 i.e., Chun method (CM1) [equation (1.1)], Ostrovski's method (OS) [equation (1.3)], Argyros et. al. method (AR) [equation (1.4)] and Shah and Noor's method (SN) [equation (1.7)]. In table-2, we present a comparative study of newly proposed Algorithm 7 (RM2) with standard Newton's method (NM) and some known methods with convergence order 5 i.e. Chun's method (CM2) [equation (1.2)], Javidi's method (JM) [equation (1.5)] and Rafiullah's method (FM) [equation (1.6)].

These comparative studies obviously reveal the better performance of our proposed methods.

We use Maple18 for all numerical computations, using the command Digits $:=300$. In Table 1 and Table $2, x^{*}$ is the approximation, obtained by using Newton's method, to the exact solution such that $\left|f\left(x^{*}\right)\right|<10^{-200}$, nit is the number of iterations performed and $n f e$ is the total number of new function evaluations. 
Table 1.

\begin{tabular}{|c|c|c|c|c|c|c|}
\hline$f(x)$ & $x_{\circ}$ & Method & nit & $\left|f\left(x_{n}\right)\right|$ & $\left|x_{n}-x^{*}\right|$ & $n f e$ \\
\hline \multirow{8}{*}{$\begin{array}{l}x^{2} \sin x-\cos x \\
x^{*}=0.8952060453 \ldots\end{array}$} & \multirow{8}{*}{1.29} & $N M$ & 6 & $1.148090 e-45$ & $4.286122 e-46$ & 12 \\
\hline & & $C M 1$ & 4 & $2.110531 e-134$ & $7.879164 e-135$ & 12 \\
\hline & & $O S$ & 4 & $1.410078 e-163$ & $5.264192 e-164$ & 12 \\
\hline & & $A R$ & 4 & $4.833224 e-167$ & $1.804369 e-167$ & 12 \\
\hline & & $S N, \beta=0.6$ & 3 & $1.081350 e-56$ & $4.036963 e-57$ & 12 \\
\hline & & $S N, \beta=0.4$ & 3 & $1.362152 e-65$ & $5.085272 e-66$ & 12 \\
\hline & & $R M 1, \beta=0.6$ & 3 & $6.490981 e-63$ & $2.423253 e-63$ & 12 \\
\hline & & $R M 1, \beta=0.4$ & 3 & $5.196603 e-65$ & $1.940028 e-65$ & 12 \\
\hline \multirow{8}{*}{$\begin{array}{l}\sin x-\frac{x}{3} \\
x^{*}=2.2788626600 \ldots\end{array}$} & \multirow{8}{*}{1.95} & $N M$ & 6 & $1.313574 e-51$ & $1.335341 e-51$ & 12 \\
\hline & & $C M 1$ & 4 & $8.085075 e-123$ & $8.219050 e-123$ & 12 \\
\hline & & $O S$ & 4 & $3.824786 e-188$ & $3.888165 e-188$ & 12 \\
\hline & & $A R$ & 4 & $3.624362 e-189$ & $3.684420 e-189$ & 12 \\
\hline & & $S N, \beta=0.6$ & 3 & $2.150035 e-73$ & $2.185663 e-73$ & 12 \\
\hline & & $S N, \beta=0.5$ & 3 & $5.931834 e-79$ & $6.030129 e-79$ & 12 \\
\hline & & $R M 1, \beta=0.6$ & 3 & $1.607844 e-74$ & $1.634487 e-74$ & 12 \\
\hline & & $R M 1, \beta=0.5$ & 3 & $1.329024 e-80$ & $1.351047 e-80$ & 12 \\
\hline \multirow{8}{*}{$\begin{array}{l}e^{x}-3 x^{2} \\
x^{*}=0.9100075724 \ldots\end{array}$} & \multirow{8}{*}{1.35} & $N M$ & 6 & $5.172517 e-47$ & $1.738250 e-47$ & 12 \\
\hline & & $C M 1$ & 4 & $6.601319 e-140$ & $2.218406 e-140$ & 12 \\
\hline & & $O S$ & 4 & $3.884077 e-170$ & $1.305263 e-170$ & 12 \\
\hline & & $A R$ & 4 & $2.604410 e-171$ & $8.752249 e-172$ & 12 \\
\hline & & $S N, \beta=0.6$ & 3 & $1.469888 e-64$ & $4.939632 e-65$ & 12 \\
\hline & & $S N, \beta=0.4$ & 3 & $3.456924 e-63$ & $1.161716 e-63$ & 12 \\
\hline & & $R M 1, \beta=0.6$ & 3 & $5.072980 e-65$ & $1.704800 e-65$ & 12 \\
\hline & & $R M 1, \beta=0.4$ & 3 & $2.106149 e-63$ & $7.077816 e-64$ & 12 \\
\hline \multirow{8}{*}{$\begin{array}{l}x^{2}-(1-x)^{5} \\
x^{*}=0.3459548158 \ldots\end{array}$} & \multirow{8}{*}{0.9} & $N M$ & 6 & $3.238285 e-33$ & $2.015279 e-33$ & 12 \\
\hline & & $C M 1$ & 4 & $1.608446 e-109$ & $1.000982 e-109$ & 12 \\
\hline & & OS & 4 & $6.551806 e-93$ & $4.077379 e-93$ & 12 \\
\hline & & $A R$ & 4 & $1.195335 e-86$ & $7.438916 e-87$ & 12 \\
\hline & & $S N, \beta=0.6$ & 3 & $2.139363 e-45$ & $1.331388 e-45$ & 12 \\
\hline & & $S N, \beta=0.4$ & 3 & $7.849351 e-66$ & $4.884878 e-66$ & 12 \\
\hline & & $R M 1, \beta=0.6$ & 3 & $2.255151 e-37$ & $1.403446 e-37$ & 12 \\
\hline & & $R M 1, \beta=0.4$ & 3 & $5.931836 e-87$ & $3.691553 e-87$ & 12 \\
\hline \multirow{8}{*}{$\begin{array}{l}x^{3}+4 x^{2}-15 \\
x^{*}=1.6319808055 \ldots\end{array}$} & \multirow{8}{*}{1.1} & $N M$ & 6 & $1.028368 e-35$ & $4.886301 e-37$ & 12 \\
\hline & & $C M 1$ & 4 & $8.246965 e-64$ & $3.918556 e-65$ & 12 \\
\hline & & $O S$ & 4 & $4.081933 e-158$ & $1.939535 e-159$ & 12 \\
\hline & & $A R$ & 4 & $4.081933 e-158$ & $1.939535 e-159$ & 12 \\
\hline & & $S N, \beta=0.6$ & 3 & $1.310591 e-56$ & $6.227290 e-58$ & 12 \\
\hline & & $S N, \beta=0.5$ & 3 & $1.160337 e-62$ & $5.513355 e-64$ & 12 \\
\hline & & $R M 1, \beta=0.6$ & 3 & $2.080529 e-68$ & $9.885659 e-70$ & 12 \\
\hline & & $R M 1, \beta=0.5$ & 3 & $4.023494 e-64$ & $1.911768 e-65$ & 12 \\
\hline \multirow{8}{*}{$\begin{array}{l}e^{-x}+\sin x-x \\
x^{*}=1.2349822792 \ldots\end{array}$} & \multirow{8}{*}{2} & $N M$ & 6 & $7.277483 e-42$ & $7.570443 e-42$ & 12 \\
\hline & & $C M 1$ & 4 & $8.211356 e-125$ & $8.541910 e-125$ & 12 \\
\hline & & $O S$ & 4 & $2.756393 e-192$ & $2.867354 e-192$ & 12 \\
\hline & & $A R$ & 4 & $4.770000 e-197$ & $4.960000 e-197$ & 12 \\
\hline & & $S N, \beta=0.5$ & 3 & $1.377560 e-55$ & $1.433014 e-55$ & 12 \\
\hline & & $S N, \beta=0.4$ & 3 & $2.851880 e-56$ & $2.966684 e-56$ & 12 \\
\hline & & $R M 1, \beta=0.5$ & 3 & $1.438312 e-59$ & $1.496212 e-59$ & 12 \\
\hline & & $R M 1, \beta=0.4$ & 3 & $4.734088 e-59$ & $4.924662 e-59$ & 12 \\
\hline \multirow{8}{*}{$\begin{array}{l}\arctan x+e^{x} \\
x^{*}=-0.6065554097 \ldots\end{array}$} & & $N M$ & 6 & $1.693915 e-38$ & $1.327241 e-38$ & 12 \\
\hline & & $C M 1$ & 4 & $3.983685 e-76$ & $3.121354 e-76$ & 12 \\
\hline & & $O S$ & 4 & $1.062625 e-181$ & $8.326028 e-182$ & 12 \\
\hline & -11 & $A R$ & 4 & $2.870544 e-184$ & $2.249170 e-184$ & 12 \\
\hline & -1.1 & $S N, \beta=0.6$ & 3 & $2.565503 e-58$ & $2.010160 e-58$ & 12 \\
\hline & & $S N, \beta=0.5$ & 3 & $8.621490 e-64$ & $6.755233 e-64$ & 12 \\
\hline & & $R M 1, \beta=0.6$ & 3 & $5.333099 e-73$ & $4.178666 e-73$ & 12 \\
\hline & & $R M 1, \beta=0.5$ & 3 & $2.873472 e-65$ & $2.251464 e-65$ & 12 \\
\hline & & $N M$ & 6 & $2.020829 e-37$ & $7.197216 e-38$ & 12 \\
\hline & & $C M 1$ & 4 & $1.326213 e-74$ & $4.723331 e-75$ & 12 \\
\hline & & $O S$ & 4 & $1.151457 e-106$ & $4.100934 e-107$ & 12 \\
\hline$e^{x}+\cos \pi x$ & -1.75 & $A R$ & 4 & $1.201507 e-107$ & $4.279189 e-108$ & 12 \\
\hline$x^{*}=-1.4224873831 \ldots$ & -1.75 & $S N, \beta=0.6$ & 3 & $8.533065 e-62$ & $3.039066 e-62$ & 12 \\
\hline & & $S N, \beta=0.4$ & 3 & $2.270015 e-47$ & $8.084698 e-48$ & 12 \\
\hline & & $R M 1, \beta=0.6$ & 3 & $2.784886 e-70$ & $9.918420 e-71$ & 12 \\
\hline & & $R M 1, \beta=0.4$ & 3 & $1.241340 e-52$ & $4.421052 e-53$ & 12 \\
\hline
\end{tabular}


Table 2.

\begin{tabular}{|c|c|c|c|c|c|c|}
\hline$f(x)$ & $x_{\circ}$ & Method & nit & $\left|f\left(x_{n}\right)\right|$ & $\left|x_{n}-x^{*}\right|$ & $n f e$ \\
\hline \multirow{6}{*}{$\begin{array}{l}\sin ^{2} x-x^{2}+1 \\
x^{*}=1.4044916482 \ldots\end{array}$} & \multirow{6}{*}{1.8} & $N M$ & 6 & $3.854345 e-40$ & $1.552623 e-40$ & 12 \\
\hline & & $C M 2$ & 3 & $7.501114 e-75$ & $3.021630 e-75$ & 12 \\
\hline & & $J M$ & 3 & $2.958821 e-71$ & $1.191884 e-71$ & 15 \\
\hline & & $F M$ & 3 & $5.253392 e-65$ & $2.116193 e-65$ & 15 \\
\hline & & $R M 2, \beta=0.6$ & 3 & $7.078690 e-142$ & $2.851467 e-142$ & 12 \\
\hline & & $R M 2, \beta=0.4$ & 3 & $2.684536 e-76$ & $1.081396 e-76$ & 12 \\
\hline \multirow{6}{*}{$\begin{array}{l}(x+2) e^{x}-1 \\
x^{*}=-0.4428544010 \ldots\end{array}$} & \multirow{6}{*}{-0.77} & $N M$ & 6 & $3.098223 e-39$ & $1.886629 e-39$ & 12 \\
\hline & & $C M 2$ & 3 & $8.476683 e-76$ & $5.161782 e-76$ & 12 \\
\hline & & $J M$ & 3 & $7.092642 e-64$ & $4.318986 e-64$ & 15 \\
\hline & & $F M$ & 3 & $5.361171 e-50$ & $3.264626 e-50$ & 15 \\
\hline & & $R M 2, \beta=0.6$ & 3 & $1.549638 e-102$ & $9.436350 e-103$ & 12 \\
\hline & & $R M 2, \beta=0.7$ & 3 & $8.337059 e-127$ & $5.076760 e-127$ & 12 \\
\hline \multirow{6}{*}{$\begin{array}{l}e^{x}-3 x^{2}-3 \\
x^{*}=3.8695271180 \ldots\end{array}$} & \multirow{6}{*}{3.7} & $N M$ & 6 & $6.200279 e-51$ & $2.509974 e-52$ & 12 \\
\hline & & $C M 2$ & 3 & $3.445765 e-98$ & $1.394902 e-99$ & 12 \\
\hline & & $J M$ & 3 & $5.238746 e-89$ & $2.120730 e-90$ & 15 \\
\hline & & $F M$ & 3 & $5.622100 e-77$ & $2.275918 e-78$ & 15 \\
\hline & & $R M 2, \beta=0.6$ & 3 & $5.909145 e-99$ & $2.392119 e-100$ & 12 \\
\hline & & $R M 2, \beta=0.7$ & 3 & $1.160571 e-111$ & $4.698181 e-113$ & 12 \\
\hline \multirow{6}{*}{$\begin{array}{l}x^{3}-10 \\
x^{*}=2.1544346900 \ldots\end{array}$} & \multirow{6}{*}{1.7} & $N M$ & 6 & $1.364324 e-38$ & $9.797822 e-40$ & 12 \\
\hline & & $C M 2$ & 3 & $1.411484 e-71$ & $1.013650 e-72$ & 12 \\
\hline & & $J M$ & 3 & $1.091787 e-63$ & $7.840610 e-65$ & 15 \\
\hline & & $F M$ & 3 & $1.234363 e-49$ & $8.864514 e-51$ & 15 \\
\hline & & $R M 2, \beta=0.6$ & 3 & $5.752944 e-147$ & $4.131447 e-148$ & 12 \\
\hline & & $R M 2, \beta=0.4$ & 3 & $5.261890 e-87$ & $3.778800 e-88$ & 12 \\
\hline \multirow{6}{*}{$\begin{array}{l}x^{3}+x^{2}-2 \\
x^{*}=1.0000000000 \ldots\end{array}$} & \multirow{6}{*}{1.4} & $N M$ & 6 & $2.109328 e-37$ & $4.218655 e-38$ & 12 \\
\hline & & $C M 2$ & 3 & $4.001294 e-72$ & $8.002588 e-73$ & 12 \\
\hline & & $J M$ & 3 & $2.425961 e-66$ & $4.851922 e-67$ & 15 \\
\hline & & $F M$ & 3 & $3.138825 e-60$ & $6.277649 e-61$ & 15 \\
\hline & & $R M 2, \beta=0.6$ & 3 & $1.153387 e-86$ & $2.306774 e-87$ & 12 \\
\hline & & $R M 2, \beta=0.7$ & 3 & $1.946779 e-119$ & $3.893557 e-120$ & 12 \\
\hline \multirow{6}{*}{$\begin{array}{l}e^{x}-4 x^{2} \\
x^{*}=0.7148059123 \ldots\end{array}$} & \multirow{6}{*}{1.15} & $N M$ & 6 & $1.026736 e-38$ & $2.794101 e-39$ & 12 \\
\hline & & $C M 2$ & 3 & $1.712603 e-70$ & $4.660578 e-71$ & 12 \\
\hline & & $J M$ & 3 & $1.070387 e-68$ & $2.912891 e-69$ & 15 \\
\hline & & $F M$ & 3 & $1.125335 e-62$ & $3.062423 e-63$ & 15 \\
\hline & & $R M 2, \beta=0.6$ & 3 & $2.358759 e-102$ & $6.418991 e-103$ & 12 \\
\hline & & $R M 2, \beta=0.4$ & 3 & $7.757362 e-78$ & $2.111044 e-78$ & 12 \\
\hline \multirow{6}{*}{$\begin{array}{l}x^{3}+4 x^{2}-10 \\
x^{*}=1.3652300134 \ldots\end{array}$} & \multirow{6}{*}{1} & $N M$ & 6 & $3.982349 e-43$ & $2.411587 e-44$ & 12 \\
\hline & & $C M 2$ & 3 & $6.364581 e-80$ & $3.854192 e-81$ & 12 \\
\hline & & $J M$ & 3 & $4.080246 e-73$ & $2.470870 e-74$ & 15 \\
\hline & & $F M$ & 3 & $6.930588 e-60$ & $4.196948 e-61$ & 15 \\
\hline & & $R M 2, \beta=0.6$ & 3 & $1.543351 e-135$ & $9.346053 e-137$ & 12 \\
\hline & & $R M 2, \beta=0.7$ & 3 & $4.464117 e-123$ & $2.703330 e-124$ & 12 \\
\hline \multirow{6}{*}{$\begin{array}{l}x^{2}-(1-x)^{5} \\
x^{*}=0.3459548158 \ldots\end{array}$} & \multirow{6}{*}{0.9} & $N M$ & 6 & $3.238285 e-33$ & $2.015279 e-33$ & 12 \\
\hline & & $C M 2$ & 3 & $5.042834 e-50$ & $3.138302 e-50$ & 12 \\
\hline & & $J M$ & 3 & $3.939960 e-59$ & $2.451951 e-59$ & 15 \\
\hline & & $F M$ & 3 & $3.333525 e-54$ & $2.074549 e-54$ & 15 \\
\hline & & $R M 2, \beta=0.6$ & 3 & $7.956289 e-88$ & $4.951429 e-88$ & 12 \\
\hline & & $R M 2, \beta=0.4$ & 3 & $5.675379 e-101$ & $3.531952 e-101$ & 12 \\
\hline \multirow{6}{*}{$\begin{array}{l}e^{x}-3 x^{2} \\
x^{*}=0.9100075724 \ldots\end{array}$} & \multirow{6}{*}{0.63} & $N M$ & 6 & $3.578716 e-43$ & $1.202645 e-43$ & 12 \\
\hline & & $C M 2$ & 3 & $7.310670 e-73$ & $2.456787 e-73$ & 12 \\
\hline & & $J M$ & 3 & $2.283532 e-72$ & $7.673921 e-73$ & 15 \\
\hline & & $F M$ & 3 & $1.848608 e-58$ & $6.212337 e-59$ & 15 \\
\hline & & $R M 2, \beta=0.6$ & 3 & $2.243660 e-95$ & $7.539930 e-96$ & 12 \\
\hline & & $R M 2, \beta=0.7$ & 3 & $3.188270 e-113$ & $1.071434 e-113$ & 12 \\
\hline
\end{tabular}

\section{CONCLUSIONS}

In the present paper, we develop two new iterative methods with a parameter for solving nonlinear equations. The orders of convergence of our proposed methods are four and five and thus the efficiency indices are $4^{\frac{1}{4}} \approx 1.41421356$ and $5^{\frac{1}{4}} \approx 1.49534878$, respectively. Comparison of our methods with standard Newton's method and some other known methods with the same convergence orders is presented in Table 1-2.

In terms of the test functions, the initial values, the parameters used and the $n f e$, it is obvious that our 4th order method $R M 1$ gives better results than the methods $N M$ and $S N$, but the methods $C M 1, O S$ and $A R$ give better results than our method $R M 1$. On the other hand, our 5 th order method $R M 2$ outperforms all the other 5 th order methods used in the comparison. 
The idea and technique used in this paper can be employed to develop and analyze higher order multi-step iterative methods for solving nonlinear equations. Finally, determination of possible effective auxiliary functions $g(x)$ other than $g(x)=e^{-\beta x}$ is a problem that worths investigation.

\section{Acknowledgement}

The authors are grateful to the referee for his valuable comments and helpful suggestions which proved to be a source of improved version of the paper.

\section{References}

[1] S. Abbasbandy, Improving Newton-Raphson method for nonlinear equations by modified Adomian decomposition method, Appl. Math. Comput. 145 (2003) 887-893.

[2] I.K. Argyros, D. Chen, Q. Qian, The Jarrat method in Banach space setting, J. Comput. Appl. Math. 51 (1994) 103-106.

[3] G. Adomian, Nonlinear Stochastic system and applications to physics, Kluwer Academic Publishers, Dordrecht, 1989.

[4] E. Babolian and J. Biazar, Solution of nonlinear equations by modified Adomian decomposition method, Appl. Math. Comput. 132 (2002), 167-172.

[5] C. Chun, Construction of Newton-like iterative methods for solving nonlinear equations, Numer. Math. 104 (2006) 297-315.

[6] V. Daftardar-Gejji, H. Jafari, An iterative method for solving nonlinear functional equations, J. Anal. 316 (2006), 753-763.

[7] A. Golbabai, M. Javidi, A third-order Newton type method for nonlinear equations based on modified homotopy perturbation method, Appl. Math. Comput. 191(2007), 199-205.

[8] Y. Ham, C. Chun, A fifth order iterative method for solving nonlinear equations, Appl. Math. Comput. 194(2007), 287-290

[9] J. H. He, A new iteratration method for solving algebraic equations, Appl. Math. Comput. 135 (2003) 81-84.

[10] J. H. He, Homotopy perturbation technique, Comput. Methods Appl. Mech. Eng. 178(3-4) (1999), 257-262.

[11] J. H. He, A coupling method of a homotopy technique and a perturbation technique for non-linear problems, Int. J. Non-Linear Mech. 35(1) (2000), 37-43.

[12] J. H. He, The homotopy perturbation method for non-linear oscillators with discontinuities, Appl. Math. Comput. 151(2004), 287-292.

[13] J. H. He, Application of homotopy perturbation method to nonlinear wave equations, Chaos Solitons Fractals 26(3) (2005), 695-700.

[14] J. H. He, Asymptotology by homotopy perturbation method, Appl. Math. Comput. 156(3) (2004), 591-596.

[15] J. H. He, Homotopy perturbation method for solving boundary problems, Phys. Lett. A. $350(1-2)$ (2006), 87-88.

[16] J. H. He, Limit cycle and bifurcation of nonlinear problems, Chaos Solitons Fractals 26(3) (2005), 827-833.

[17] J. H. He, Variational iteration method-some recent results and new interpretations, J. Appl. Math. Comput. 207 (2007) 3-17.

[18] M. Javidi, Fourth-order and fifth-order iterative methods for nonlinear algebraic equations, Math. Comput. Modelling 50 (2009) 66-71.

[19] M. A. Noor, New Classes of iterative methods for nonlinear equations, Appl. Math. Comput. 191 (2007) 128-131.

[20] A.M. Ostrowski, Solution of equations and system of equations, Academic press, New York, 1966.

[21] M. Rafiullah, A Fifth-order Iterative Method for Solving Nonlinear Equations, Sibirskii Zhurnal Vychislitel'noi Mathematiki. 14(3) (2011), 297-302.

[22] F.A. Shah, M.A. Noor, Some numerical methods for solving nonlinear equations by using decomposition technique, Appl. Math. Comput. 251 (2015), 378-386. 
[23] J.F. Traub, Iterative Methods for the Solution of Equations, Prentice-Hall Englewood Cliffs, New Jersey, USA, 1964. 
